\title{
Post-Pandemic Transformation of Healthcare Delivery in Provinces and Territories
}

S I write this, positive signs regarding the pandemic are beginning to
affect our daily lives. We are now able to meet with our extended families, friends
and neighbours. For many people, these changes signal the end of isolation, fear and loneliness and harken a return to normal life and behaviours.

Changes are now also appearing in the healthcare sector. Long-term care homes have been reopened to families and visitors. Homecare visits are returning to their pre-pandemic levels, and hospitals have restarted all elective surgeries.

We should celebrate the fact that the delivery of acute and emergency healthcare carried on throughout the pandemic. With some exceptions, cancer-related care continued throughout, trauma care was available and dialysis continued. This occurred even as the focus of governments, organizations and individual providers shifted to the pandemic's effects on physical and mental health.

Many new policies, procedures and physical spaces were used to deliver healthcare during the COVID-19 pandemic in order to provide critical care and lessen the risks of transmission within healthcare organizations and among providers, patients, their families and the community. As reopening unfolds, some of the significant challenges facing the federal government, provinces and territories are these: What new practices and behaviours should be retained in the post-pandemic era? How will the changes be paid for?

\section{Competing Options for Healthcare Delivery}

It is not yet clear how the pandemic has impacted current federal, provincial and territorial healthcare budgets. While healthcare is already a significant component of provinces' and territories' economies, the guess is that healthcare spending has jumped substantively.

For the healthcare sector, the short-term focus will be on provincial and territorial governments' actions and what they signal as their new-normal state for healthcare delivery-- 
I think that the policy-making window is open and many important decisions regarding healthcare delivery are going to be made in the near-term. In the interim, provincial and territorial governments, their ministers and deputy ministers of health will be the recipients of competing views on healthcare policy from the public, advocacy groups, government experts and health system researchers. For instance, one policy trade-off for which provincial governments could solicit input includes the following: Should long-term care be redesigned or should provinces and territories focus on reducing surgical backlogs caused by the COVID-19 pandemic? Both options are important, very expensive and compete for the same healthcare budget.

Emerging from a pandemic, the public voice will be particularly raw. This includes longterm care residents and families that have endured exceptionally difficult circumstances. The voices of patients and families should, and will, shape some governments' budgetary priorities for healthcare services.

Advocacy groups also provide provincial and territorial governments with input into potential changes in healthcare services and policy. Many advocacy groups play an important role in the health sector by representing oft-overlooked subgroups particularly disadvantaged by the pandemic. Their input ranges from minor policies - such as proposing new physician billing codes - to more major and expensive policies, such as staff's skill sets and scopes of practice in long-term care homes.

In some provinces, governments have nurtured in-house expertise in health system analyses. This pool of expertise will be critical in providing governments with spending and utilization models and projections. These outputs will constitute options for post-pandemic health policies. However, provinces or territories that have hollowed their internal expertise over the years will find themselves needing help and will need to either hire consultancy services or risk making expensive mistakes.

Lastly, health systems research is also used as one input into the government's healthcare policy options. However, careful research often requires years of data collection and interpretation - a lag that is too long to inform contemporaneous post-pandemic policy making and spending decisions. Complicating this perspective is that many health systems researchers have followed the money and pivoted to COVID-related research themes, thereby lessening the pool of available talent to generate non-COVID-related policy research.

\section{Strengthening Long-Term Outcomes}

New post-pandemic healthcare policies will have important consequences for healthcare delivery and spending. For each change in healthcare delivery models, the federal, provincial or territorial governments will have to pay for new treatment modalities and healthcare facilities or codify COVID-induced changes to practice. Good spending decisions by governments will maximize the value of new spending each policy generates. Poor investments may saddle provinces and territories with legacy programs and services that they cannot rid themselves of, thereby robbing value from future healthcare services. 
To reduce the risk of poor investments, there are practical - and relatively inexpensive steps that can be taken. Comparative data regarding the value of competing policies are needed: What is the health and quality of life that will be generated? Whom do the benefits go to? What are the costs and what other contextual information is needed to inform the government's decisions? This information may transcend traditional age- and sex-based analyses and encompass new budgetary analyses that include gains in employment status or situational vulnerability.

To generate these key insights to inform government policy makers, two key ingredients are needed: data and expertise. When it comes to the former, comprehensive health and social care data should be made more available as soon as possible with fewer encumbrances as currently exist. Regarding the latter, federal, provincial and territorial governments should target funding to policy-relevant health systems research as soon as possible.

The availability of comprehensive and timely data and the engagement of experts are needed to provide evidence-informed options for governments and complement other channels of input. As the window for policy change is at least partially open, this is the time for federal and provincial government action. Federal, provincial and territorial government investments are needed to support health systems research whose objectives are to provide policy options that avoid costly mistakes.

\section{In This Issue}

This issue of Healthcare Policy is led by a Discussion and Debate article addressing the COVID-19-induced challenges of engaging with patients. The article by Sayani et al. (2021) describes how the pandemic put a halt to the health system's engagement with patients, arguing that the health system's reopening provides an opportunity to focus on patientidentified priorities and a diversity of perspectives. For researchers and policy makers, the authors conclude by listing a number of attributes to use for engaging with socially marginalized groups and reducing health inequalities.

The Discussion and Debate article is followed by a rejoinder enhancing the discussion of the role of patients in engagement and partnership. Shaw et al. (2021) contend that the perspective of patients alone is not sufficient; the article posits that the perspectives of local communities are needed to provide input into the structure and processes of their health systems. The rejoinder concludes by advocating that "communities" should co-lead health system design in order to reduce inequities in health.

A second Discussion and Debate article focuses on the issue of medical malpractice insurance in provinces and territories. The article explains that the Canadian Medical Protective Association (CMPA) is a fund primarily dedicated to defending physicians against patients' and their families' lawsuits. Describing how the provinces and territories subsidize the CMPA premiums used to defend physicians, Lee et al. (2021) note that less than $0.3 \%$ of claimants have ever received compensation. The article concludes by advocating for significant reforms to the tort-based medical malpractice system. 
Echoing the structural inefficiencies and expenses of the current system articulated by Lee et al. (2021), the rejoinder authored by Nelson (2021) adds to the topic of medical malpractice reform. This article argues that a no-fault system as a replacement would likely improve access to compensation, but would not address underlying issues of safety, appropriateness or accountability in healthcare, which are important to many patients. The article concludes that the current tort-based system needs reform, though further exploration of incentives including blended models may lead to a preferable balance between patient compensation and physician accountability.

In a Data Matters article, Aggarwal et al. (2021) describe the attributes of family physician data across provinces and territories. The authors detail that there is no national source of family physician data that includes the distribution of or comprehensively describes the practice characteristics of family physicians. The authors note that while there are over 20 sources of information on family physicians, none are sufficiently comprehensive or granular to support detailed workforce planning or detailed health systems research. The article concludes that strong federal leadership, support and funding are needed to develop a reliable data infrastructure for family physicians, complementary to other pan-Canadian data standards.

In Research Papers, Ogundeji et al. (2021) present the results of an Alberta-based, qualitative study of specialist physician incentives and payment models. As physician compensation models have remained static for decades, with some minor exceptions, this study reported on respondents' perspectives regarding alternative models. The results showed that blended fee-for-service and salary-based models may be more preferable to the health system in order to improve the value of physician services - when accompanied with suitable accountability mechanisms to measure value and quality. The study concludes that blended remuneration models are feasible for specialist physicians, and possibly preferred by specialists; however, legislative changes and new partnerships are required.

The next article in Research Papers presents the results and implications of the rapid adoption of telehealth in primary care clinics in Quebec during the COVID-19 pandemic. Based on an anonymous cross-sectional survey of teaching primary care clinics, Breton et al. (2021) reported that telephone-based consultations experienced the most significant increases in utilization, exceeding video consultations. The authors also reported that telephoneand video-based consultations limited the ability to collect relevant clinical information, potentially causing duplication of tests or services. The article concludes by identifying a number of important issues left to be addressed by policy makers, including telehealth training for physicians or allied healthcare providers and potential inequities in patients' access to technologies.

The focus of the third article in Research Papers is alternative level of care in Ontario. Alternative level of care is a perennial problem for all provinces' hospitals, with many hospitalized patients occupying beds that do not need the technologies or skilled care provided 
in acute care. Written by Nauenberg (2021), the article discusses the feasibility of Ontario following other jurisdictions in making use of empty residential spaces to temporarily house alternative level of care patients. Describing Ontario's regulatory, training and policy barriers to moving in this direction, the article concludes that novel health services and policy approaches are needed for the serious problem of alternative level of care.

The final article of this issue describes the implementation of the hub-and-spoke model for coordinated and integrated care for medically complex children in Ontario. Authored by Lin et al. (2021), this qualitative study of an implementation model reported strengthened communication between acute, specialty and community care, and improved family relationships. However, the study also reported challenges in overcoming fragmentation and resourcing dedicated social care workers, nursing support or mental health providers. The article concludes with a number of recommendations for other provinces to improve health and social care for medically complex children.

JASON M. SUTHERLAND, PHD

Editor-in-Chief

\section{References}

Aggarwal, M., A. Katz and I. Oandasan. 2021. Current State of Quantitative Data Available for Examining the Work of Family Physicians in Canada. Healthcare Policy 17(1): 48-57. doi:10.12927/hcpol.2021.26578.

Breton, M., N. Deville-Stoetzel, I. Gaboury, M.A. Smithman, J. Kaczorowski, M.-T. Lussier et al. 2021. Telehealth in Primary Healthcare: A Portrait of its Rapid Implementation during the COVID-19 Pandemic. Healthcare Policy 17(1): 73-90. doi:10.12927/hcpol.2021.26576.

Lee, S.K., B.H. Rowe, C.M. Flood and S.K. Mahl. 2021. Canada's System of Liability Coverage in the Event of Medical Harm: Is It Time for No-Fault Reform? Healthcare Policy 17(1): 30-41. doi:10.12927/ hcpol.2021.26580.

Lin, J.L.L., S. Quartarone, N. Aidarus, C.Y. Chan, J. Hubbert, J. Orkin et al. 2021. Process Evaluation of a Hub-and-Spoke Model to Deliver Coordinated Care for Children with Medical Complexity across Ontario: Facilitators, Barriers, and Lessons Learned. Healthcare Policy 17(1): 104-22. doi:10.12927/hcpol.2021.26574.

Nauenberg, E. 2021. Personal Support Homes: An Innovative Approach to Reduce the Alternative Level of Care Population in Ontario Hospitals. Healthcare Policy 17(1): 91-103. doi:10.12927/hcpol.2021.26575.

Nelson, E.L. 2021. Commentary: Some Questions about No-Fault Reform of the Medical Liability System. Healthcare Policy 17(1): 42-47. doi:10.12927/hcpol.2021.26579.

Ogundeji, Y.K., A. Quinn, M. Lunney, C. Chong, D. Chew, G. Hopkin et al. 2021. Optimizing Physician Payment Models to Address Health System Priorities: Perspectives from Specialist Physicians. Healthcare Policy 17(1): 58-72. doi:10.12927/hcpol.2021.26577.

Sayani, A., A. Maybee, J. Manthorne, E. Nicholson, G. Bloch, J.A. Parsons et al. 2021. Building Equitable Patient Partnerships during the COVID-19 Pandemic: Challenges and Key Considerations for Research and Policy. Healthcare Policy 17(1): 17-24. doi:10.12927/hcpol.2021.26582.

Shaw, J., P. Sky and S. Chandra. 2021. Commentary: Community Knowledge for Equity in Healthcare. Healthcare Policy 17(1): 25-29. doi:10.12927/hcpol.2021.26581. 


\section{Transformation de la prestation des soins de santé dans les provinces et les territoires après la pandémie}

U MOMENT D'ÉCRIRE CES Lignes, LeS Signes Positifs CONCERNANT LA PANDÉmie
commencent à se faire sentir sur notre quotidien. Nous pouvons maintenant visiter
famille élargie, amis et voisins. Pour plusieurs, ces changements marquent la fin de l'isolement, de la peur et de la solitude et annoncent un retour à une vie plus normale.

Des changements se font aussi voir dans le secteur de la santé. Les foyers de soins de longue durée sont rouverts aux familles et aux visiteurs. La prestation de soins à domicile revient au niveau qui prévalait avant la pandémie et les hôpitaux ont repris toutes les chirurgies non urgentes.

Il faut souligner le fait que la prestation de soins de courte durée et de soins d'urgence se soit poursuivie tout au long de la pandémie. À quelques exceptions près, on a pu maintenir en place les soins liés au cancer, les soins de traumatologie et la dialyse. Tout cela alors même que les gouvernements, les organisations et les travailleurs de la santé devaient affronter les effets de la pandémie sur la santé physique et mentale.

Plusieurs nouvelles politiques, procédures et lieux ont été mis à contribution pour fournir des soins intensifs et réduire les risques de transmission au sein des organisations de soins de santé et parmi les prestataires, les patients, leurs familles et la communauté. Avec la réouverture en cours, le gouvernement fédéral, les provinces et les territoires font face à d'importants défis. Quelles nouvelles pratiques et comportements devraient être conservés après la pandémie? Comment ces changements seront-ils payés?

\section{Choix concurrents pour la prestation des services de santé}

On ne sait pas encore clairement quel est l'impact de la pandémie sur les budgets de santé fédéraux, provinciaux et territoriaux. Bien que les soins de santé constituent déjà une composante importante de l'économie des provinces et territoires, on suppose que les dépenses en santé ont considérablement augmenté. 
Dans le secteur de la santé, l'accent à court terme sera mis sur l'action des gouvernements provinciaux et territoriaux et ce qu'ils indiquent comme la nouvelle normalité dans la prestation des soins de santé.

Je pense quune fenêtre s'ouvre pour l'élaboration de politiques et que de nombreuses décisions importantes concernant la prestation des soins de santé seront prises à court terme. Dans l'intervalle, les gouvernements provinciaux et territoriaux ainsi que les ministres et sous-ministres de la Santé devront faire face à des points de vue divergents de la part de la population, des groupes de défense, des experts gouvernementaux et des chercheurs du système de santé. Les gouvernements provinciaux pourraient solliciter des commentaires sur la question politique suivante : les provinces et territoires doivent-ils repenser les soins de longue durée ou plutôt se concentrer sur la réduction des retards chirurgicaux causés par la pandémie de COVID-19? Les deux points de la question sont importants, très coûteux et se disputent le même budget de santé.

$\mathrm{Au}$ sortir de la pandémie, la voix de la population sera particulièrement acerbe. Pensons notamment aux résidents des foyers de soins de longue durée et aux familles qui ont enduré des circonstances particulièrement difficiles. Leur voix devrait éclairer les priorités budgétaires en matière de santé chez certains gouvernements.

Les groupes de défense fournissent également aux gouvernements provinciaux et territoriaux des commentaires sur les changements potentiels dans les services et les politiques de santé. De nombreux groupes jouent un rôle important dans le secteur de la santé en représentant des sous-groupes souvent négligés et particulièrement défavorisés par la pandémie. Leurs commentaires vont de politiques secondaires - telles que la proposition de nouveaux codes de facturation des médecins - à des politiques plus importantes et coûteuses, telles que l'ensemble des compétences du personnel ou les champs d'exercice des foyers de soins de longue durée.

Les gouvernements de certaines provinces ont développé une expertise interne pour les analyses du système de santé. Ce bassin d'expertise sera essentiel pour fournir aux gouvernements des modèles et des projections de dépenses et d'utilisation. Ces extrants peuvent éclairer les choix de politiques dans la foulée de la pandémie. Cependant, les provinces ou les territoires qui ont épuisé leur expertise interne au fil des ans auront besoin d'aide et devront avoir recours à des services de consultation, sans quoi ils risquent de commettre des erreurs coûteuses.

La recherche sur les systèmes de santé peut aussi contribuer à l'élaboration des politiques de santé. Cependant, une recherche minutieuse nécessite souvent des années de collecte et d'interprétation de données - un délai trop long pour éclairer l'élaboration de politiques et les décisions de dépenses après la pandémie. Pour compliquer le tout, de nombreux chercheurs ont suivi l'argent et se sont tournés vers des thèmes de recherche liés à la COVID-19, réduisant ainsi le bassin de talents disponibles pour mener des recherches sur les politiques non liées à la pandémie. 


\section{Renforcement des résultats dans le long terme}

Les nouvelles politiques de santé auront des conséquences importantes sur la prestation des services et les dépenses de santé. Pour chaque changement dans le modèle de prestation des soins de santé, les gouvernements fédéral, provinciaux ou territoriaux devront payer pour de nouvelles modalités de traitement et de nouveaux établissements de santé, ou devront codifier les changements de pratique induits par la COVID-19. Les bonnes décisions de dépenses maximiseront la valeur des nouvelles dépenses générées par chaque politique. De mauvais investissements peuvent accabler les provinces et les territoires de programmes et de services hérités dont ils ne peuvent se débarrasser, diminuant ainsi le poids d'éventuels services de santé.

Des mesures pratiques et relativement peu coûteuses peuvent être prises pour réduire le risque de mauvais investissements. Des données comparatives concernant la valeur des politiques concurrentes sont nécessaires : quelles type de santé et de qualité de vie seront générées? Qui en bénéficiera? Quels en seront les coûts et quelles informations contextuelles seront nécessaires pour éclairer les décisions du gouvernement? Ces informations peuvent transcender les analyses traditionnelles fondées sur l'âge et le sexe pour englober de nouvelles analyses budgétaires qui incluent des gains de statut d'emploi ou de vulnérabilité situationnelle.

Pour générer ces informations afin d'informer les décideurs, deux ingrédients clés sont nécessaires : les données et l'expertise. En effet, des données complètes sur la santé et les services sociaux devraient être disponibles le plus tôt possible avec moins de charges quéen l'état actuel. Pour ce qui est de l'expertise, les gouvernements fédéral, provinciaux et territoriaux devraient cibler le plus tôt possible le financement de recherches pertinentes pour l'élaboration de politiques.

La disponibilité de données complètes et opportunes ainsi que l'engagement d'experts sont nécessaires pour fournir aux gouvernements des choix fondés sur les données probantes et pour compléter d'autres canaux de contribution. Puisque la fenêtre du changement est au moins partiellement ouverte, c'est le moment d'agir. Les investissements des gouvernements fédéral, provinciaux et territoriaux sont nécessaires pour soutenir la recherche sur les systèmes de santé, recherche dont l'objectif est d'apporter des choix stratégiques qui permettront d'éviter les erreurs coûteuses.

\section{Dans le présent numéro}

Ce numéro de Politiques de Santé śouvre avec un article de la section Discussions et débats qui aborde les défis en matière d'engagement avec les patients dans le contexte de la COVID-19. Larticle de Sayani et al. (2021) décrit comment la pandémie a mis un terme à l'engagement du système de santé auprès des patients et affirme que la réouverture du système offre l'occasion de se concentrer sur les priorités identifiées par les patients et sur la diversité des 
points de vue. Pour les chercheurs et les décideurs, les auteurs concluent en énumérant un certain nombre de caractéristiques dont il faut tenir compte pour s'engager auprès des groupes socialement marginalisés et pour réduire les inégalités en matière de santé.

Cet article est suivi d'une réplique qui vient appuyer la discussion sur le rôle des patients dans l'engagement et les partenariats. Shaw et al. (2021) soutiennent que le seul point de vue des patients n'est pas suffisant. L'article postule que le point de vue des communautés locales est nécessaire pour contribuer à la structure et aux processus des systèmes de santé. La réplique conclut en préconisant que les « communautés » devraient codiriger la conception du système de santé afin de réduire les inégalités en matière de santé.

Un deuxième article de la section Discussions et débats porte sur la question de l'assurance contre la faute professionnelle médicale dans les provinces et les territoires. L'article explique que l'Association canadienne de protection médicale (ACPM) est un fonds principalement dédié à la défense des médecins contre les poursuites judiciaires des patients et de leurs familles. En décrivant comment les provinces et les territoires subventionnent les primes de l'ACPM utilisées pour défendre les médecins, Lee et al. (2021) constatent que moins de 0,3 \% des demandeurs reçoivent une indemnisation. L'article conclut en plaidant pour une réforme importante du système de faute professionnelle médicale fondée sur la responsabilité délictuelle.

Faisant écho aux inefficacités structurelles et aux dépenses du système décrites par Lee et al. (2021), la réplique rédigée par Nelson (2021) concerne aussi la réforme au sujet des fautes professionnelles médicales. Cet article postule qu'un système de remplacement sans égard à la responsabilité améliorerait probablement l'accès à l'indemnisation, mais ne résoudrait pas les problèmes sous-jacents en matière de sécurité, de pertinence ou de responsabilité des soins de santé, lesquels sont importants pour de nombreux patients. L'article conclut que le système actuel fondé sur la responsabilité délictuelle doit être réformé, bien qu'une exploration plus poussée des incitatifs, y compris les modèles mixtes, puisse conduire à un équilibre souhaitable entre l'indemnisation des patients et la responsabilité des médecins.

Dans un article de la section Questions de données, Aggarwal et al. (2021) décrivent la nature des données sur les médecins de famille dans les provinces et les territoires. Les auteurs précisent qu'il n'existe pas de source nationale de données qui comprend la distribution ou qui décrit de manière exhaustive les caractéristiques de la pratique des médecins de famille. Les auteurs notent que bien qu'il existe plus de 20 sources d'information sur les médecins de famille, aucune n'est suffisamment complète ou précise pour soutenir une planification détaillée de la main-d'œuvre ou une recherche détaillée sur les systèmes de santé. Larticle conclut qu'un leadership, un soutien et un financement vigoureux de la part du gouvernement fédéral seront nécessaires pour développer une infrastructure de données fiables, complémentaire aux autres normes de données pancanadiennes.

Dans la section Rapports de recherche, Ogundeji et al. (2021) présentent les résultats d'une étude qualitative sur les incitatifs et les modèles de rémunération des médecins 
spécialistes en Alberta. Puisque les modèles de rémunération des médecins sont demeurés statiques pendant des décennies, à quelques petites exceptions près, cette étude rend compte du point de vue des répondants concernant les modèles alternatifs. Les résultats ont montré que les modèles mixtes de rémunération à l'acte et de salaire peuvent être préférables pour le système de santé afin d'améliorer la valeur des services médicaux, lorsqu'ils s'accompagnent de mécanismes de responsabilisation appropriés pour en mesurer la valeur et la qualité. L'étude conclut que les modèles de rémunération mixte sont réalisables, et possiblement préférés, par les spécialistes; cependant, des modifications législatives et de nouveaux partenariats sont nécessaires.

L'article suivant dans la section Rapports de recherche présente les résultats et les répercussions de l'adoption rapide de la téléconsultation dans les cliniques de soins primaires au Québec pendant la pandémie de COVID-19. Sur la base d'une enquête transversale anonyme auprès de cliniques universitaires de soins primaires, Breton et al. (2021) signalent que les consultations par téléphone ont connu l'augmentation la plus importante, dépassant les consultations par vidéo. Les auteurs signalent aussi que les consultations par téléphone et par vidéo limitent la capacité de collecter des informations cliniques pertinentes, ce qui peut entraîner une duplication des tests ou des services. Larticle conclut en identifiant un certain nombre de problèmes importants que les décideurs doivent aborder, notamment la formation en télésanté pour les médecins et les prestataires de soins ainsi que les inégalités potentielles concernant l'accès aux technologies pour les patients.

Le troisième article de la section Rapports de recherche met l'accent sur les autres niveaux de soins en Ontario. Les jours d'autres niveaux de soins constituent un problème permanent pour les hôpitaux de toutes les provinces, avec de nombreux patients hospitalisés occupant des lits, mais qui n'ont pas besoin des technologies ou des soins qualifiés fournis en courte durée. Rédigé par Nauenberg (2021), l'article examine la possibilité que l'Ontario suive l'exemple d'autres autorités sanitaires en utilisant des espaces résidentiels vacants pour héberger temporairement les patients d'un autre niveau de soins. Tout en décrivant les obstacles en matière de réglementation, de formation ou de politique qui empêchent l'Ontario d'aller en ce sens, l'article conclut que des services de santé novateurs et de nouvelles approches politiques seront nécessaires pour résoudre le grave problème des autres niveaux de soins.

Le dernier article de ce numéro décrit la mise en œuvre du modèle en étoile pour des soins coordonnés et intégrés pour les enfants qui nécessitent des soins complexes en Ontario. Rédigé par Lin et al. (2021), cette étude qualitative d'un modèle de mise en œuvre fait état d'une communication renforcée entre les soins de courte durée, les soins spécialisés et les services communautaires. L'étude signale aussi une amélioration des relations familiales. Cependant, l'étude fait aussi état de difficultés à surmonter la fragmentation et à trouver des ressources pour les travailleurs sociaux, le soutien infirmier ou les prestataires de soins de 
santé mentale. L'article se termine par un certain nombre de recommandations à l'intention des autres provinces qui souhaitent améliorer les soins de santé et les services sociaux pour les enfants qui présentent une complexité médicale.

\author{
JASON M. SUTHERLAND, PHD \\ Rédacteur en chef
}

\title{
Références
}

Aggarwal, M., A. Katz et I. Oandasan. 2021. État actuel des données quantitatives disponibles pour examiner le travail des médecins de famille au Canada. Politiques de Santé 17(1): 48-57. doi:10.12927/hcpol.2021.26578.

Breton, M., N. Deville-Stoetzel, I. Gaboury, M.A. Smithman, J. Kaczorowski, M.-T. Lussier et al. 2021. Téléconsultations de première ligne : portrait d'une mise en œuvre rapide pendant la pandémie de COVID-19. Politiques de Santé 17(1): 73-90. doi:10.12927/hcpol.2021.26576.

Lee, S.K., B.H. Rowe, C.M. Flood et S.K. Mahl. 2021. Système canadien de couverture de responsabilité en cas de préjudice médical : le temps est-il venu de procéder à une réforme sans égard à la responsabilité? Politiques de Santé 17(1): 30-41. doi:10.12927/hcpol.2021.26580.

Lin, J.L.L., S. Quartarone, N. Aidarus, C.Y. Chan, J. Hubbert, J. Orkin et al. 2021. Évaluation du processus d'un modèle en étoile pour offrir des soins coordonnés aux enfants présentant une complexité médicale en Ontario : facilitateurs, obstacles et leçons apprises. Politiques de Santé 17(1): 104-22. doi:10.12927/ hcpol.2021.26574.

Nauenberg, E. 2021. Foyers de soins personnels : une approche novatrice pour réduire la population d'autres niveaux de soins dans les hôpitaux ontariens. Politiques de Santé 17(1): 91-103. doi:10.12927/hcpol.2021.26575.

Nelson, E.L. 2021. Commentaire : quelques questions sur la réforme sans égard à la responsabilité dans le régime de responsabilité médicale. Politiques de Santé 17(1): 42-47. doi:10.12927/hcpol.2021.26579.

Ogundeji, Y.K., A. Quinn, M. Lunney, C. Chong, D. Chew, G. Hopkin et al. 2021. Optimiser les modèles de rémunération des médecins pour répondre aux priorités du système de santé : point de vue des médecins spécialistes. Politiques de Santé 17(1): 58-72. doi:10.12927/hcpol.2021.26577.

Sayani, A., A. Maybee, J. Manthorne, E. Nicholson, G. Bloch, J.A. Parsons et al. 2021. Mise en place de partenariats équitables avec les patients pendant la pandémie de COVID-19 : défis et considérations pour la recherche et l'élaboration de politiques. Politiques de Santé 17(1): 17-24. doi:10.12927/hcpol.2021.26582.

Shaw, J., P. Sky et S. Chandra. 2021. Commentaire : connaissances communautaires pour l'équité dans les soins de santé. Politiques de Santé 17(1): 25-29. doi:10.12927/hcpol.2021.26581. 